\title{
Insights into steroidogenic acute regulatory protein (StAR)-dependent cholesterol transfer in mitochondria: evidence from molecular modeling and structure-based thermodynamics supporting the existence of partially unfolded states of StAR
}

\author{
A P Mathieu, A Fleury, L Ducharme, P Lavigne ${ }^{1}$ and J-G LeHoux \\ Department of Biochemistry, Faculty of Medicine, Université de Sherbrooke, Quebec, Canada J1H 5N4 \\ ${ }^{1}$ Department of Pharmacology, Faculty of Medicine, Université de Sherbrooke, Quebec, Canada J1H 5N4 \\ (Requests for offprints should be addressed to J-G LeHoux or P Lavigne; Email: jean.guy.lehoux@usherbrooke.ca or pierre.lavigne@usherbrooke.ca)
}

\begin{abstract}
The steroidogenic acute regulatory protein (StAR) is the major entrance for cholesterol in mitochondria under acute stimulation. Under such circumstances, dysfunctional StAR activity can ultimately lead to lipoid congenital adrenal hyperplasia (LCAH). A complete understanding of the StAR's molecular structure and mechanism is essential to comprehend LCAH. Thus far, there is no mechanistic model that can explain experimental results at the molecular level. This is partly due to the lack of the molecular structure of StAR. The closest approximation to the StAR molecular structure is the human MLN64 which has a similar activity to StAR, has a highly homologous primary structure and for which an X-ray structure is known. In this context, we have modeled the structure of StAR through standard homology modeling procedures based on the MLN64 structure. Our StAR model shows the presence of a hydrophobic cavity of $783.9 \AA^{2}$ in surface area, large enough to fit one molecule of cholesterol. In addition, we have identified a unique charged pair, as in MLN64, lining the surface of the cavity and which could play a key role in the binding of cholesterol through the formation of an $\mathrm{H}$-bond with its $\mathrm{OH}$ moiety. This suggests that the cholesterol-binding site of StAR is located inside this cavity. Taking into account that internal cavities are destabilizing to native protein structures and that the lining of the cavity has to become accessible in order to allow cholesterol binding, we have explored the possibility that StAR could exist in equilibrium with partially unfolded states. Using a structure-based thermodynamics approach, we show that partially folded states (with an unfolded C-terminal $\alpha$-helix, and an open cavity) can be significantly populated at equilibrium and therefore allow cholesterol binding. These results are supported by recent experiments that show a loss of StAR helical character upon binding of an analog of cholesterol. Moreover, we show that the replacement of the residues involved in the charged-pair located in the binding site results in the loss of StAR activity, supporting a key role for these residues. Taken together, our results are applicable to StAR functioning both in the mitochondrial intermembrane space as well as outside the mitochondria.
\end{abstract}

Journal of Molecular Endocrinology (2002) 29, 327-345

\section{Introduction}

Until recently, it was believed that the ratelimiting step in steroid hormone synthesis was the conversion of cholesterol into pregnenolone by the cytochrome $\mathrm{P} 450$ side-chain cleavage ( $\mathrm{P} 450$ scc) enzyme in the inner membrane of mitochondria (Farkash et al. 1986). However, it was discovered that the true rate-limiting step in steroidogenesis is the transfer of cholesterol from the outer mitochondrial membrane, through the aqueous intermembrane space, to the inner mitochondrial membrane where the P450 scc resides (Brownie et al. 1972, Simpson et al. 1978, Crivello \& Jefcoate 1980, Privalle et al. 1983). 
As potential cholesterol transferring proteins, the best candidate to date is the steroidogenic acute regulatory protein (StAR) first cloned and characterized in the MA-10 mouse Leydig tumor cell line by Clark et al. (1994). Effectively, these authors were the first to identify StAR as being the same $30-\mathrm{kDa}$ protein originally described by OrmeJohnson and colleagues (Pon et al. 1986, Pon \& Orme-Johnson 1988, Alberta et al. 1989, Epstein \& Orme-Johnson 1991a,b, Hartigan et al. 1995), responsible for adrenocorticotropin hormone (ACTH)-stimulated steroidogenesis. The involvement of StAR in cholesterol translocation is supported by a number of experimental results, the most obvious being the StAR knockout mice (Caron et al. 1997) displaying lipoid congenital adrenal hyperplasia (LCAH). Patients with LCAH cannot synthesize adequate amounts of steroids and are characterized by cholesterol accumulation in the adrenal glands and gonads. This potentially lethal condition is now commonly attributed to a dysfunctional StAR, most importantly in the critical C-terminal region, as demonstrated by StAR mutants (Lin et al. 1995, Bose et al. 1996). In humans, StAR is expressed in adrenal glands, gonads, and brain, and consists of 285 amino acids (Sugawara et al. 1995) separated into two functional domains: (1) the mitochondrial import sequence responsible for its localization in mitochondria, and (2) the StAR lipid transfer (START) domain (Ponting \& Aravind 1999, Ponting et al. 1999) responsible for the transfer of cholesterol into the inner membrane of mitochondria.

START domains have been discovered and characterized for a large variety of proteins with different functions (Homma \& Emori 1995, Masucci et al. 1996, Ponting \& Aravind 1999, Ponting et al. 1999, Raya et al. 1999). Of these, the human MLN64 protein may have a similar function as the human StAR in the placenta, brain, and non-steroidogenic tissues for the formation of steroids and bile acids (Bieche et al. 1996, Moog-Lutz et al. 1997, Watari et al. 1997). The structure of MLN64 was recently solved by X-ray crystallography and revealed several interesting features (Tsujishita \& Hurley 2000). More importantly, the cholesterol binding site is best described as a hydrophobic tunnel formed predominantly from a U-shaped unclosed $\beta$-barrel topped by the parallel C-terminal $\alpha$-helix. The tunnel completely traverses the entire MLN64 and has been suggested to work as an intermembrane shuttle (Tsujishita \& Hurley 2000), although the exact mechanism by which StAR mediates cholesterol transfer is still a subject of intensive research. Several mechanisms have been suggested for the action of cholesterol transfer by StAR. These include desorption, the implication of a molten globule state and the intermembrane shuttle (Stocco \& Clark 1996, Stocco 2000, 2001).

Desorption requires that the inner and outer mitochondrial membranes come into contact as StAR is imported into the mitochondrial matrix by its mitochondrial targeting sequence (Kallen et al. 1998). Then, high volumes of cholesterol can be transferred from the cholesterol-rich outer membrane to the cholesterol-depleted inner membrane. This mechanism accounts for the localization of mitochondrial-imported proteins at contact points (Schwaiger et al. 1987, Pon et al. 1989, Stocco \& Clark 1996), the rapid cholesterol transfer requiring little StAR, and the quick degradation of StAR by mitochondria (Clark et al. 1994, Stocco \& Clark 1996). It does not however, account for the fact that StAR can still function without its N-terminal import sequence (Arakane et al. 1996, 1998). As for the molten globule state (Christensen et al. 2001, Bose et al. 2002), it was shown that StAR loses tertiary structure at low $\mathrm{pH}(3 \cdot 5-4 \cdot 0)$ while retaining secondary structures, suggesting that partially folded states of StAR are populated at low $\mathrm{pH}$ and even at $\mathrm{pH}$ values closer to neutrality (Christensen et al. 2001). In this study, the authors suggest that this 'molten globular state' is important for StAR function outside mitochondria (Bose et al. 2002). This model is in agreement with the observation that an active electrochemical force is required for the transfer of cholesterol (King et al. 1999) but the in vivo local acidic environment still remains to be proven. Finally, the intermembrane shuttle states that StAR acts as a sterol carrier, migrating between mitochondrial membranes (Tsujishita \& Hurley 2000). The molecular mechanism and structural requirement for the carrier role of StAR is still elusive. On the other hand, it appears that the structure of MLN64 may reveal key features to help understand the mode of cholesterol transport by StAR. The X-ray structure of MLN64 (Tsujishita \& Hurley 2000) depicts a hydrophobic tunnel characterized by $\beta$-strands forming the floor and sidewalls, topped by a C-terminal $\alpha$-helix and two loops. Inside the 
tunnel, there is an opening of the cavity such that one cholesterol molecule may fit. On the other hand, the tunnel openings are not large enough to allow a cholesterol molecule to pass through StAR. Tsujishita and Hurley (2000) suggest that transient conformational changes of the loops and/or the C-terminal $\alpha$-helix allow cholesterol access to the hydrophobic tunnel, a view supported by the recent crystallization of the phosphatidylinositol transfer protein $\alpha$ (PITP $\alpha)$ open conformation (Schouten et al. 2002); PITP $\alpha$ is involved in the regulation of lipid and vesicular trafficking and in lipid-mediated signal transduction pathways (Cunningham et al. 1995, Cockcroft 1999). Importantly, in support of the C-terminal $\alpha$-helix transient conformational changes, it has recently been observed that there is loss of helical content in StAR upon binding of a cholesterol chromophoric analog, NBD-cholesterol (Petrescu et al. 2001).

Following the observations that (1) the C-terminal region of StAR is important for proper StAR activity (Lin et al. 1995, Bose et al. 1996), (2) the C-terminal region is suggested to transiently change conformation for cholesterol binding (Tsujishita \& Hurley 2000), (3) the loss of StAR helical structure is obtained upon the binding of a cholesterol analog (Petrescu et al. 2001), (4) molten globular states of StAR are possible (Bose et al. 1999, Christensen et al. 2001), and (5) the C-terminal $\alpha$-helix in MLN64 entirely covers the putative cholesterol binding site, we investigated the possible implication of various conformational states of this $\mathrm{C}$-terminal $\alpha$-helix in the binding of cholesterol to StAR through molecular modeling.

We show that the hamster StAR model has a hydrophobic cavity instead of a tunnel. This cavity is proposed to be the driving force for the unfolding of the C-terminal $\alpha$-helix revealing the cholesterol binding site. We calculate through structure-based thermodynamics (Freire 1993, Lavigne et al. 2000) that this partially folded state could be significantly populated and that cholesterol favorably binds to it. We also observe that once cholesterol has bound to StAR, adjacent loops to the opened StAR binding site (loop $\Omega 1$ and the loop between $\beta$-strands 1 and 2) effectively inhibit the C-terminal $\alpha$-helix reformation, supporting the observed loss of $\alpha$-helical structure upon cholesterol binding to StAR, thus allowing a rapid cholesterol transfer inside mitochondria. Finally, we show that a peculiar salt-bridge in the binding site plays a key role in cholesterol binding. This is supported by new mutagenesis results showing that cholesterol transfer can be abolished by removal of this salt-bridge.

Taken together, these results suggest a rapid unfolded StAR mechanism for the transfer of cholesterol from the outer to the inner mitochondial membranes, driven by the transmembrane cholesterol gradient; our calculations are applicable to a StAR functioning both in the mitochondrial intermembrane space and in the cytosol in the proximity of mitochondria. Thus, we propose a plausible common ground for the intermembrane shuttle and molten globule mechanisms.

\section{Materials and methods}

\section{Modeling methodology}

All calculations were performed on an SGI Octane 2 workstation and the InsightII (2000) suite of programs (Homology, Discover, Biopolymer and Builder, Accelrys, San Diego, CA, USA) to obtain all models. The amino acid sequences for the hamster and human StAR were obtained from Genbank (Accession Numbers AAB06763 and NP_000340 respectively) and aligned to the extracted amino acid sequence of MLN64 by a CLUSTAL W algorithm (Thompson et al. 1994), and manually adjusted in Homology. The amino acid side chains of the hamster and human StAR were replaced in the MLN64 coordinate file (PDB ID number 1EM2.). The minimization calculations were performed in the absence of Coulombic potential, cross terms and morse potentials. The consistent valence forcefield (GVFF) (DauberOsguthorpe et al. 1988) was used for all calculations. Essentially, the minimization was completed in four steps: (1) fixed heavy (all but hydrogens) atoms, (2) fixed backbone atoms with tethered heavy atoms, (3) tethered backbone atoms, and (4) no atoms fixed. Each step was performed as follows: (1) steepest descent for 1000 iterations or until the maximum derivative is less than $0.001 \mathrm{kcal} / \AA$, followed by (2) conjugate gradient for 1000 iterations or until the maximum derivative is less than $0.001 \mathrm{kcal} / \AA$.

To define the cavity, we filled it with water; water molecules were discovered in the MNL64 crystal (Tsujishita \& Hurley 2000). First, a $50 \AA$ 
solvent layer was added to the StAR models, upon which the cavity was placed 'over' the water molecules. All the water molecules outside the cavity volume were removed, keeping only the water molecules that fit in the cavity. These water molecules were visually identified by comparing the molecular surfaces of the water molecules as well as the cavity.

For binding studies, a cholesterol molecule was created using the Builder module in the InsightII suite. The initial planar structure was minimized using steepest descent for 1000 iterations or until the maximum derivative was less than $0.001 \mathrm{kcal} /$ $\AA$, fixing nothing and using no Coulombic potential, cross terms and morse potentials. Thereafter, the cholesterol molecule was manually and visually placed inside the StAR cavity, as previously suggested (Tsujishita \& Hurley 2000). Due to the shape of StAR's cavity, placement of cholesterol was unambiguous. The structures were then minimized as mentioned above. The cholesterol-free native hamster and human models have been deposited in the protein data bank (PDB) and have PDB ID numbers 1 ILJ and 1 IMG respectively.

\section{Free energy calculations}

In order to calculate the Gibb's free energy difference $(\Delta G)$ between the native and partially unfolded states of StAR, we have used an approach called structure-based thermodynamics (Freire 1993) as implemented in the computer program STC (Lavigne et al. 2000). This approach has been shown to allow for the identification of partially folded (unfolded) states of proteins in equilibrium with their native states and to allow for the prediction of binding free energy of ligands to proteins (Hilser \& Freire 1997, Baker \& Murphy 1998).

Briefly, we manually unfolded the C-terminal $\alpha$-helix of the hamster StAR model using the Biopolymer module of the InsightII suite. As a model for the unfolded state, we have used an extended conformation ( $\psi$ and $\varphi=180^{\circ}$ ). Then, the changes in accessible surface area $(\triangle \mathrm{ASA})$ between the 'unfolded states' and the native state were calculated using the CALC_ASA module of STC (Lavigne et al. 2000) and separated into polar (N and $\mathrm{O}$ ) and non-polar $(\mathrm{C}$ and $\mathrm{S})$ atoms. Using the calculated $\triangle \mathrm{ASA}$ and empirical functions relating changes in heat capacity $(\Delta \mathrm{Cp})$, enthalpy $(\Delta \mathrm{H})$, and entropy $(\Delta \mathrm{S})$ per unit of $\Delta \mathrm{ASA}, \Delta \mathrm{G}$ between the different states were calculated using the THERMO module of STG (Lavigne et al. 2000). Note that no changes in the protonation states were considered. We also explored loop movement above the opened StAR cavity using this same methodology. Finally, the binding constant between cholesterol and the partially unfolded state was calculated as described elsewhere (Lavigne et al. 2000).

\section{Rendering}

All the model graphical images were created using either InsightII for molecular surfaces or Ribbons $3 \cdot 0$ for ribbon diagrams.

\section{Materials for site-directed mutagenesis}

All restriction and modifying enzymes were purchased from New England Biolabs (Mississauga, ON, Canada). The expression vector pcDNA3 1 Myc-His, lipofectamine and oligonucleotides were purchased from Invitrogen Canada Inc. (Burlington, ON, Canada). All vectors were amplified in XL1 Blue E. coli competent cells (Stratagene, La Jolla, CA, USA), and purified on Qiagen anion-exchange columns (Qiagen, Chatsworth, CA, USA). 12-Otetradecanoyl phorbol-13-acetate (TPA) and $(\mathrm{Bu})_{2}$ cAMP (cAMP) were obtained from Sigma-Aldrich (St-Louis, MO, USA).

\section{Hamster StAR mutagenesis by PCR}

Briefly, two series of PCR amplifications were obtained using the following conditions: hot start of 5 min at $94{ }^{\circ} \mathrm{C}$ (without DNA polymerase) followed by a touch down of $2{ }^{\circ} \mathrm{C}$ from $94{ }^{\circ} \mathrm{C} / 70{ }^{\circ} \mathrm{C} / 72{ }^{\circ} \mathrm{C}$ to $94{ }^{\circ} \mathrm{C} / 50{ }^{\circ} \mathrm{C} / 72{ }^{\circ} \mathrm{C}$, completed with 30 cycles of $94{ }^{\circ} \mathrm{C} / 50{ }^{\circ} \mathrm{C} / 72^{\circ} \mathrm{C}$ and a final $72{ }^{\circ} \mathrm{C}$ elongation of $15 \mathrm{~min}$. The first series consists of amplifying hamster StAR cDNA in two different reactions: first, a wild type (WT) upstream (sense) oligonucleotide to the downstream mutant (antisense) oligonucleotide at the site of interest for mutagenesis, and secondly a mutant sense oligonucleotide from the site of interest for mutagenesis to a WT antisense oligonucleotide. The second series consists of pooling both PCRs previously obtained, purified by agarose gel electrophoresis, and 
amplifying using WT $5^{\prime}$ and $3^{\prime}$ oligonucleotides. WT-StAR and all mutants were cloned into pcDNA3.1 Myc-His using unique EcoRI and HindIII restriction sites, and entirely sequenced between these restriction sites using a T7 sequencing kit from Amersham Pharmacia Biotech (Baie d'Urfé, QC, Canada), amplified, and purified on Qiagen anion-exchange columns.

\section{Hamster StAR expression}

Either WT or mutated StAR was transiently transfected into monkey kidney COS-1 cells (American Type Culture Collection, Rockville, MD, USA) using the lipofectamine method. Twenty-four hours before transfection the cells were harvested with pancreatin-EDTA solution (Invitrogen Canada Inc.) and plated at an initial density of $3.5 \times 10^{5}$ cells per $10 \mathrm{~cm}^{2}$ well in 6well plates. Cells were cultured in DMEM supplemented with $10 \%$ fetal bovine serum (FBS), 5.96 g/l HEPES, 2.2 g/1 NaHCO $3,1 \mathrm{mM}$ L-glutamine, $100 \mathrm{IU} / \mathrm{ml}$ penicillin, and $100 \mu \mathrm{g} / \mathrm{ml}$ streptomycin sulfate. DMEM containing no FBS and no antibiotics was used for transfections. Each transfection assay received $1 \mu \mathrm{g}$ DNA $(500 \mathrm{ng} / \mathrm{ml}$ F2 construct (Harikrishna et al. 1993) containing cytochrome $\mathrm{P} 450$ scc/adrenodoxin reductase/ adrenodoxin and $500 \mathrm{ng} / \mathrm{ml} \operatorname{pcDNA} 3 \cdot 1+$ pcDNA3 $\cdot 1-S t A R)$ and $10 \mu \mathrm{g} / \mathrm{ml}$ lipofectamine. Twenty-two hours after transfection cells were rinsed with PBS. Cells were then incubated for $24 \mathrm{~h}$ in DMEM without phenol red containing $10 \%$ dextran-coated charcoal-treated FBS and antibiotics without or with $(\mathrm{Bu})_{2}$-cAMP. Media were kept for pregnenolone determination and cells for immunoblotting analyses.

\section{Measurement of the hamster StAR activity}

In this study, StAR activity is defined as the quantity of pregnenolone formed by transfected COS-1 cells in co-transfection experiments using F2 (Harikrishna et al. 1993) and StAR plasmids. Pregnenolone was analyzed by radioimmunoassay (ICN Pharmaceuticals, Diagnostics Division, Orangeburg, NY, USA). This conversion of cholesterol into pregnenolone is directly related to the rate of cholesterol transfer by StAR to P450 scc in the inner mitochondrial membrane.

\section{Immunoblotting analysis}

SDS-PAGE was performed with $20 \mu \mathrm{g}$ soluble proteins on a $12 \%$ gel and analyzed by immunoblotting (LeHoux et al. 1992) using a rabbit polyclonal anti-mouse StAR antibody (kindly given by Dr Dale Buchanan Hales, Department of Physiology and Biophysics, University of Illinois at Chicago, Chicago, IL, USA) as previously described (LeHoux et al. 1996). This immunoglobulin was raised against a GST-fusion protein from a mouse StAR cDNA from a 208-1467 bp fragment (Clark et al. 1997). Cells were scraped and directly harvested in hot $\left(100^{\circ} \mathrm{C}\right.$ ) Laemmli buffer (Laemmli 1970). All samples were then passed through a 26-gauge needle, then boiled for $10 \mathrm{~min}$ and finally centrifuged at $12000 \mathbf{g}$ for $2 \mathrm{~min}$. Soluble proteins in the supernatant were determined using the Bio-Rad Protein Assay Dye Reagent (Bio-Rad Laboratories Ltd, Mississauga, ON, Canada).

Immunoreactive proteins were detected using ECL-PLUS light emitting reagents (Amersham Pharmacia Biotech UK Ltd, Amersham Place, Little Chalfont, Bucks, UK). Autoradiograms were observed by exposing the blots to Kodax X-Omat XK films. The results were also visualized and quantified on the optical imager STORM 860 using ImageQuant software version 5·0 (Molecular Dynamics, Sunnyvale, CA, USA).

\section{Statistical analyses}

Differences between experimental groups were analyzed by ANOVA followed by Dunnett's test, using the SigmaStat program for Windows (SPSS Science, Chicago, IL, USA).

\section{Results}

\section{Overall structure of molecular models}

In order to undertake theoretical molecular modeling of proteins, a suitable alignment between a protein of known three-dimensional structure and the target protein must be obtained. In this case, the alignment of the human MLN64 with the hamster and human StAR proteins is depicted in Fig. 1 and shows a suitable identity of approximately $34 \%$ between the sequence of the hamster/human StAR and MLN64. Following the unambiguousness of the alignment, the amino acid residues of the hamster and human StAR proteins 


$\begin{array}{lrllll}\text { Hum MLN64 } & 230 & \text { SFSAQEREYI RQGKEATAVV DQILAQEENW } & \text { KFEKNNEYGD } & 269 \\ \text { Ham StAR } & 66 & \text { LYSEQELSYI QQGEVAMQKA LSILSNQEGW } & \text { KKENQQENGD } & 105 \\ \text { Hum StAR } & 67 & \text { LYSDQELAYL QQGEEAMQKA LGILSNQEGW } & \text { KKESQQDNGD } & 106\end{array}$

Hum MLN64 270 TVYTIEVPFH GKTFILKTFL PCPAELVYQE VILQPERXVL

Hum StAR 107 KVMSKVVPDV GKVFRLEVVV DQPMERLYEE LVERMEAMGE

Hum MLN64 310 WNKTVTACQI LQRVEDNTLI SYDVSAGAAG GVVSPRDFVN

Hum MLN64 350

VRRIERRRDR YLSSGIATSH SAKPPTHKYV RGENGPGGXI

Hum MLN64 39

VLKSASNPRV CTFVWILNTD LKGRLPRYLI HQSLAATXFE

Hum MLN64 430 FAFHLRQRIS ELGA

Figure 1 Alignment of the hamster and human StAR, and human MLN64 amino acid sequences used for homology modeling.

were substituted in the MLN64 coordinate file and the potential energy of the model was minimized appropriately (see Materials and methods). The stereochemical quality of the models was checked with the program PROCHEGK (Laskowski et al. 1993) and was found to be excellent.

As expected, the theoretical models for the hamster and human StAR greatly resembled the crystalline structure of the human MLN64 (Tsujishita \& Hurley 2000). As demonstrated in
Fig. 2 (only the hamster model shown), the prominent feature of the hamster StAR model is the U-shaped unclosed $\beta$-strand barrel, topped by the parallel C-terminal $\alpha$-helix. Altogether, the structure is composed of four $\alpha$-helices and nine twisted anti-parallel $\beta$-strands like the MLN64. The most significant change in the overall structure of the StAR model as compared with the human MLN64 crystal is a cholesterol binding site in the shape of an oval cavity matching the size and 
structure of cholesterol (Fig. 3A - cholesterol not shown); in MLN64, the cholesterol binding site is 'tunnel-like' (Tsujishita \& Hurley 2000). Amidst the mostly hydrophobic environment of the cavity, there are two charged amino acid residues (Glu 168-Arg187, hamster numbering) forming a salt-bridge (Fig. 3B), as described previously in MLN64 (Tsujishita \& Hurley 2000).

\section{STC calculations}

To get insight into the cholesterol transfer mechanism into the mitochondria, we attempted to calculate the change in free energy of various conformations of the hamster StAR model. STG is one of the very few methods applicable to model partially folded states that has been validated by others (Freire 1993, Hilser \& Freire 1997, Baker \& Murphy 1998, Lavigne et al. 2000). We therefore utilized STC to calculate the change in free energy of the various conformational states of our StAR model and to calculate a theoretical dissociation constant $\left(K_{\mathrm{d}}\right)$.

\section{Free energy of cavity formation}

Bearing in mind that the binding site of StAR has to become accessible and that cavities inside globular proteins destabilize the native state of proteins (Creighton 1993), we have calculated the $\Delta \mathrm{G}$ for cavity formation using STC. Moreover, the water molecules discovered in the MLN64 crystal contribute to the destabilization of the hydrophobic cavity. In order to calculate the $\Delta \mathrm{G}$, we manually calculated the ASA inside the binding site of the StAR cavity. Briefly, all atoms directly adjacent to the water molecules in the solvated model of the hamster StAR were visually identified. Then the hamster StAR model was 'cut' parallel to the oval binding site (Fig. 3C) and each piece was subjected to ASA calculations separately. $\triangle \mathrm{ASA}$ between the 'cut state' and the native state was taken to be the solvated ASA ( $\triangle \mathrm{ASA}$ non-polar $=627 \cdot 8 \AA^{2}$ and $\Delta$ ASApolar $\left.=156 \cdot 1 \AA^{2}\right)$ inside the hydrated cavity. This translates into a $\Delta \mathrm{G}$ of destabilization (cavity formation) of approximately $12 \mathrm{kcal} / \mathrm{mol}$.

\section{Partial unfolding of StAR}

It is clear that StAR with a closed binding site would have to open to allow cholesterol inside. Supporting evidence for structural changes during cholesterol binding to StAR, and during cholesterol transfer inside mitochondria include: (1) the C-terminal region of StAR is important for proper StAR activity (Lin et al. 1995, Bose et al. 1996); (2) the C-terminal region as well as the loops spatially adjacent to this region are believed to transiently change conformations for cholesterol binding (Tsujishita \& Hurley 2000); (3) molten globular states of StAR are possible (Bose et al. 1999); and (4) the C-terminal $\alpha$-helix of our StAR model entirely covers the putative cholesterol binding site as for the MLN64 crystal (Tsujishita \& Hurley 2000) and should be free for movement. Yet, the most convincing discovery supporting StAR conformational changes for proper StAR activity is the loss of StAR helical structure upon the binding of a cholesterol analog in StAR (Petrescu et al. 2001). We therefore conceptualized the opening of the binding site primarily by the movement of the G-terminal $\alpha$-helix covering the U-shaped unclosed $\beta$-barrel such as could happen in a molten globule state, driven by the $\Delta \mathrm{G}$ of destabilization caused by the hydrophobic cavity hydration as calculated above. In order to test this hypothesis, we modeled different conformations of the C-terminal $\alpha$-helix of the hamster StAR model. It is evident that any single conformation model is in effect a representation of an ensemble of conformations resulting from the dynamic nature of protein structure. Therefore, these conformational models should be seen as average structures. In the absence of cholesterol, three key conformations were considered: (A) native, (B) opened - C-terminal $\alpha$-helix still folded but removed from the binding site, and $(\mathrm{G})$ partially unfolded - G-terminal $\alpha$-helix unfolded but with the rest of the molecule native. The free energy change for all the steps is indicated in Fig. 4 (A, B, and $\mathrm{C}$ respectively). Note that we have subtracted the $\Delta \mathrm{G}$ for cavity formation from the calculated $\Delta \mathrm{G}$ change between state $\mathrm{A}$ and $\mathrm{B}$; indeed, the destabilization of the native state by the cavity has to be taken into account. The net free energy change between the native state (A) and the partially unfolded state $(\mathrm{C})$ is $+2.61 \mathrm{kcal} / \mathrm{mol}$. To a first approximation, i.e. considering the population $(\mathrm{P})$ of this state relative to the native state according to the following equation:

$$
P=\frac{\exp (-\Delta G / R T)}{1+\exp (-\Delta G / \mathrm{RT})}
$$




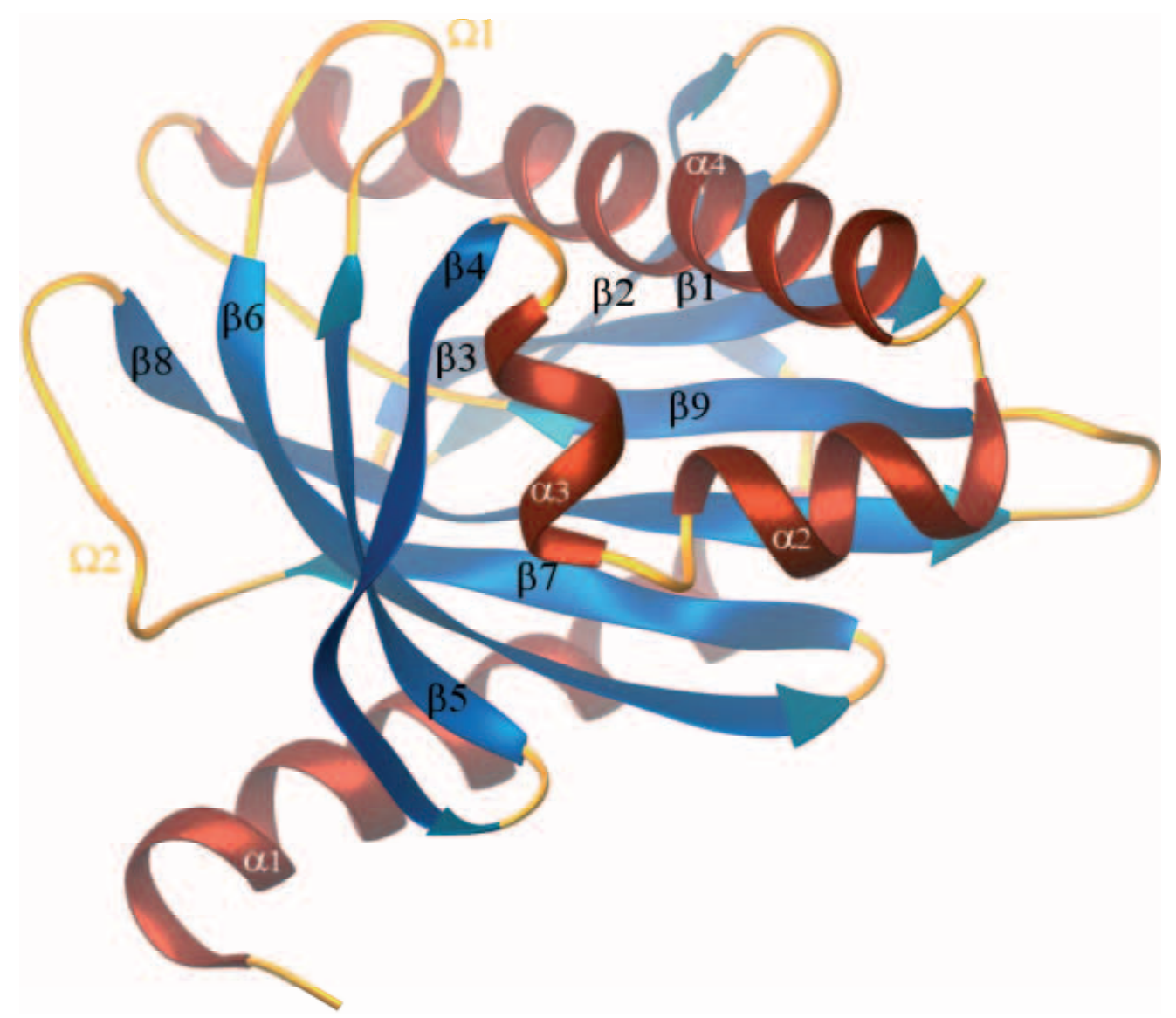

Figure 2 Ribbon diagram of the final hamster StAR model. The same overall structure was obtained for the human StAR and is therefore not shown. Helices in red, strands in blue, and loops in yellow. There is 'fog' in the image for depth perception. All structural elements have been labeled according to the human MLN64 crystal structure.

we can estimate a significant relative population of approximately $2 \%$ for this partially unfolded conformation. Note that state $\mathrm{B}$ is less stable than state $\mathrm{C}$, mostly due to the cost in conformational entropy for maintaining the helical conformation of the $\mathrm{C}$-terminal $\alpha$-helix without tertiary interactions.

\section{Binding of cholesterol to the partially unfolded state}

Next, we have calculated the $\Delta \mathrm{G}$ of binding cholesterol to the partially unfolded state of StAR using STC, as described elsewhere (Lavigne et al. 2000), and found a relatively weak $K_{\mathrm{d}}$ of $9 \cdot 44 \times 10^{-5} \mathrm{M}$ (Fig. 4, G to D). Once cholesterol binds to the partially unfolded state of the hamster StAR protein, the free energy of the complex drops below the free energy of the native state (corrected for the cost of cavity formation). Since it was discovered that there is loss of helical character upon cholesterol binding (Petrescu et al. 2001), we simulated movement of the loops adjacent to the cholesterol binding site (loop $\Omega 1$ and loop between $\beta$-strands 1 and 2) such as to inhibit the C-terminal $\alpha$-helix reformation. These loops are the only structures available for movement in the vicinity of the C-terminal $\alpha$-helix, as mentioned previously (Tsujishita \& Hurley 2000), that could aid in maintaining it in the unfolded state; Fig. 5 (D to E) depicts the movement of these loops and represents a partially refolded state of StAR. 

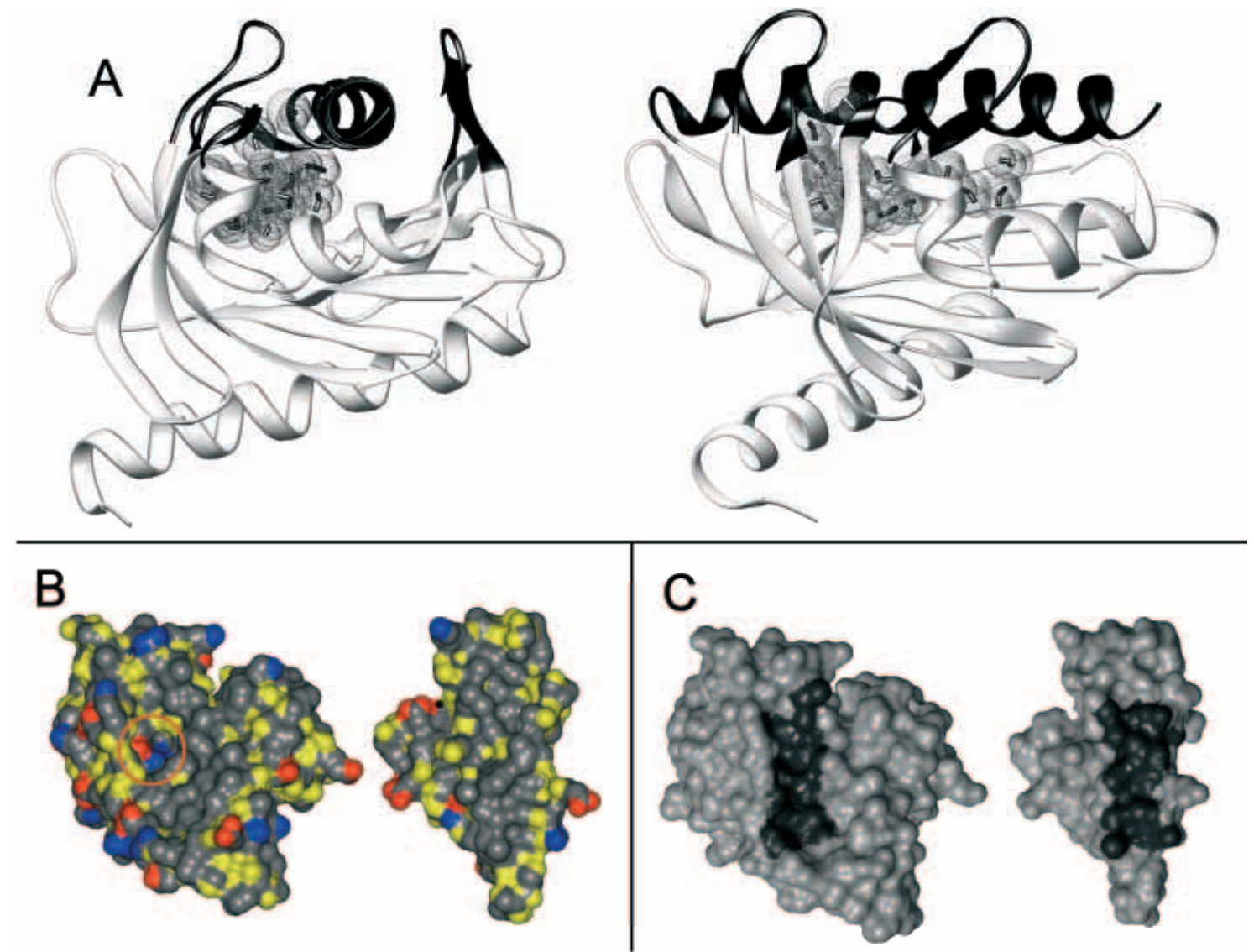

Figure 3 Hamster StAR model cholesterol binding site. (A) Ribbon diagram of the hamster StAR with a solvated binding site. The solvent is represented as Connolly surface dots with the water molecules as black sticks. The black and gray areas show where the model has been 'opened' to allow surface area calculations of the cholesterol binding site. In black, the major feature is the C-terminal $\alpha$-helix which tops the binding site; in gray, the major feature is the U-shaped $\beta$-barrel forming the bottom of the binding site, along with the majority of the remaining amino acids. (B) Connolly surface representation of the 'opened' hamster StAR model, as depicted from (A). Left, U-shaped $\beta$-barrel as seen from above the cholesterol binding site. Right, C-terminal $\alpha$-helix seen from below (as though in the cholesterol binding site). Color scheme: hydrophobic atoms in gray, potentially polar atoms in yellow, and charged atoms in red (oxygens) and blue (nitrogens). The cholesterol binding site salt-bridge is circled in orange.

(C) Same representation as in B except everything in white but the atoms directly in contact with the cholesterol binding site are in black.

\section{Trapping cholesterol inside the cavity of the StAR protein}

Although it was observed that there is loss of helical structure upon binding of cholesterol inside (Petrescu et al. 2001), it is still conceivable that the C-terminal $\alpha$-helix refolds on top of the bound cholesterol. We thus simulated the refolding of the C-terminal $\alpha$-helix, trapping cholesterol inside StAR (Fig. $6, D$ to $F$ to $G$ ). A $\Delta G$ for a closing reaction of the folded C-terminal $\alpha$-helix trapping the cholesterol inside was calculated (conformation

www.endocrinology.org
A to $\mathrm{G})$ to be $-17.91 \mathrm{kcal} / \mathrm{mol}$. As one can see, there is a dramatic stabilization of the complex. As discussed below we propose that this reaction could be involved in the shut off of the transfer.

\section{Cholesterol binding site}

As mentioned above, the cholesterol binding site of the hamster StAR model took the shape of a hydrophobic cavity and not a hydrophobic tunnel (Fig. 3). We thus investigated the possibility that water molecules could change the binding site 


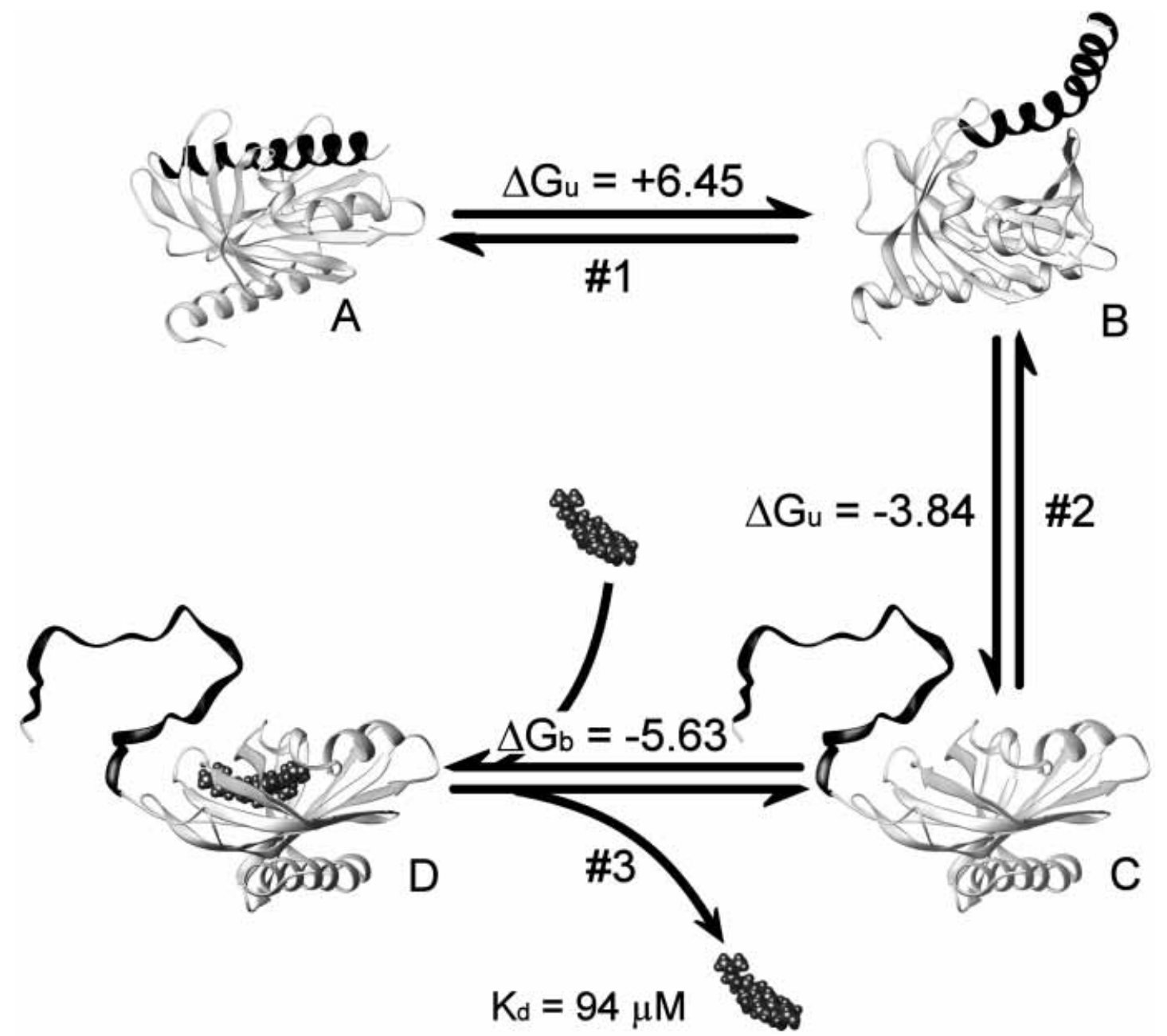

Figure 4 Molecular mechanism of cholesterol binding in StAR. The cholesterol binding site of StAR is opened through two movements of the C-terminal $\alpha$-helix (dark gray) from the original folded conformation (A): step \#1 opened (B) - completely removed from the cholesterol binding site, and step \#2 partially unfolded (C) - all of the $\alpha$-helical structure of the C-terminal $\alpha$-helix is lost. Once open, cholesterol can bind to StAR (D) as indicated in step \#3; a theoretical $K_{\mathrm{d}}$ of $94.4 \mu \mathrm{M}$ was calculated with STC. StAR is represented as a ribbons diagram while cholesterol is depicted as van der Waals radii. StAR is white with the C-terminal $\alpha$-helix black, and cholesterol dark gray. $\Delta \mathrm{G}$ is the change in Gibb's free energy in $\mathrm{kcal} / \mathrm{mol}$. $\Delta \mathrm{Gu}$, unfolding; $\Delta \mathrm{Gb}$, binding.

conformation since water molecules were discovered inside the MLN64 crystal (Tsujishita \& Hurley 2000). The model was then solvated in the binding site as well as surrounding the protein as described in Materials and methods. After minimization, the overall structure of the model and the cholesterol binding site did not alter. A cholesterol molecule was then inserted in the hamster StAR models and minimized accordingly. In the minimized complex, the $3 \beta-\mathrm{OH}$ group of cholesterol was localized adjacent to the salt- bridge formed by Glu168-Arg187 as we expected (Fig. 7). As seen, the $3 \beta-\mathrm{OH}$ group was localized $<6 \AA$ away, which is a sufficient distance for one water molecule to bridge the $3 \beta-\mathrm{OH}$ of cholesterol and the salt-bridge through hydrogen bonding.

\section{Mutant activity alterations}

The salt-bridge mutants of the hamster StAR were expressed in COS-1 cells and verified for 


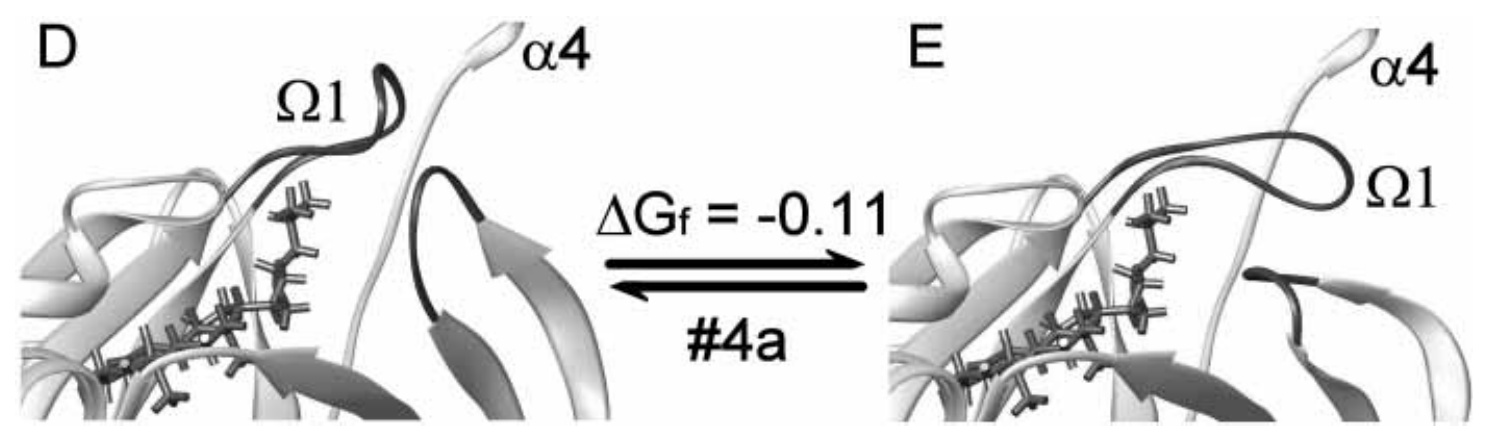

Figure 5 Representation of loop mobility upon cholesterol binding to StAR. Cholesterol binding was simulated in the partially unfolded StAR (Fig. 4D); after cholesterol binding, the loops immediately adjacent to the unfolded C-terminal $\alpha$-helix (loop $\Omega 1$ and the loop between $\beta$-strands 1 and 2) cover the bound cholesterol $(E)$, inhibiting the refolding of the C-terminal $\alpha$-helix. (D) Partially unfolded StAR (same as in Fig. 4D); (E) partially refolded StAR with loops 'closed'. Main StAR structure drawn as white ribbons with the exception of the moveable loops in dark gray; cholesterol represented as van der Waals radii, also in dark gray. $\Delta \mathrm{G}$ is the change in Gibb's free energy in kcal/mol. $\Delta \mathrm{Gf}$, folding.

alterations in activity. In both cases, when either of the amino acids involved in the formation of the salt-bridge were changed for a hydrophobic residue of similar volume (i.e. Glu168 Leu, and Arg187 Met), total loss of StAR activity was obtained in the absence of cAMP stimulation as compared with the mock transfections (Fig. 8A). Under cAMP stimulation, an increase in StAR activity was obtained but this was never higher than the non-stimulated wild type indicating a critical role for this salt-bridge in the StAR-mediated transfer of cholesterol.

\section{Mutant SDS-PAGE analysis}

Whole cell extracts from the mutant hamster StAR-expressing COS-1 cells were analyzed by SDS-PAGE (Fig. 8B). Under non-stimulated conditions, WT and mutant hamster StAR is expressed in similar proportions, as seen by the ensemble of three experiments (Fig. 8C). Under cAMP stimulation, protein concentrations were markedly increased, similarly for all preparations. The control empty vector pcDNA3.1 did not produce detectable StAR protein but a basal StAR-independent cholesterol transfer into mitochondria was obtained (Bose et al. 1996). Unexpectedly, different migratory patterns were obtained for the hamster StAR mutant Glu168 Leu (E168 L) preparations, which remain unexplained.

\section{Discussion}

\section{Molecular modeling and mutagenesis}

As expected, the structure of the hamster and human StAR models greatly resemble the human MLN64 crystal structure. The most surprising structural difference compared with the MLN64 crystal structure was the loss of the hydrophobic tunnel replaced by a hydrophobic cavity in our StAR models, matching the overall structure of cholesterol. The cavity can be imagined as the driving force allowing StAR to open, exposing the binding site to incoming cholesterol molecules. Indeed, trapping water molecules inside this hydrophobic cavity, as those co-crystallized in the MLN64 (Tsujishita \& Hurley 2000), severely reduces StAR stability. According to our structurebased thermodynamic calculations, this hydration destabilizes StAR by approximately $12 \mathrm{kcal} / \mathrm{mol}$, driving the unfolding of the $\mathrm{C}$-terminal $\alpha$-helix in solution. With a net difference of $2.61 \mathrm{kcal} / \mathrm{mol}$ between the original empty folded state and the open unfolded state of StAR, we can expect approximately $2 \%$ of the StAR population to be in the partially unfolded state.

The salt-bridge inside the cavity can be conceptualized as having two major functions: first, allowing cholesterol to bind in the proper orientation inside the well defined cavity, and secondly maintaining minimal StAR folding for 


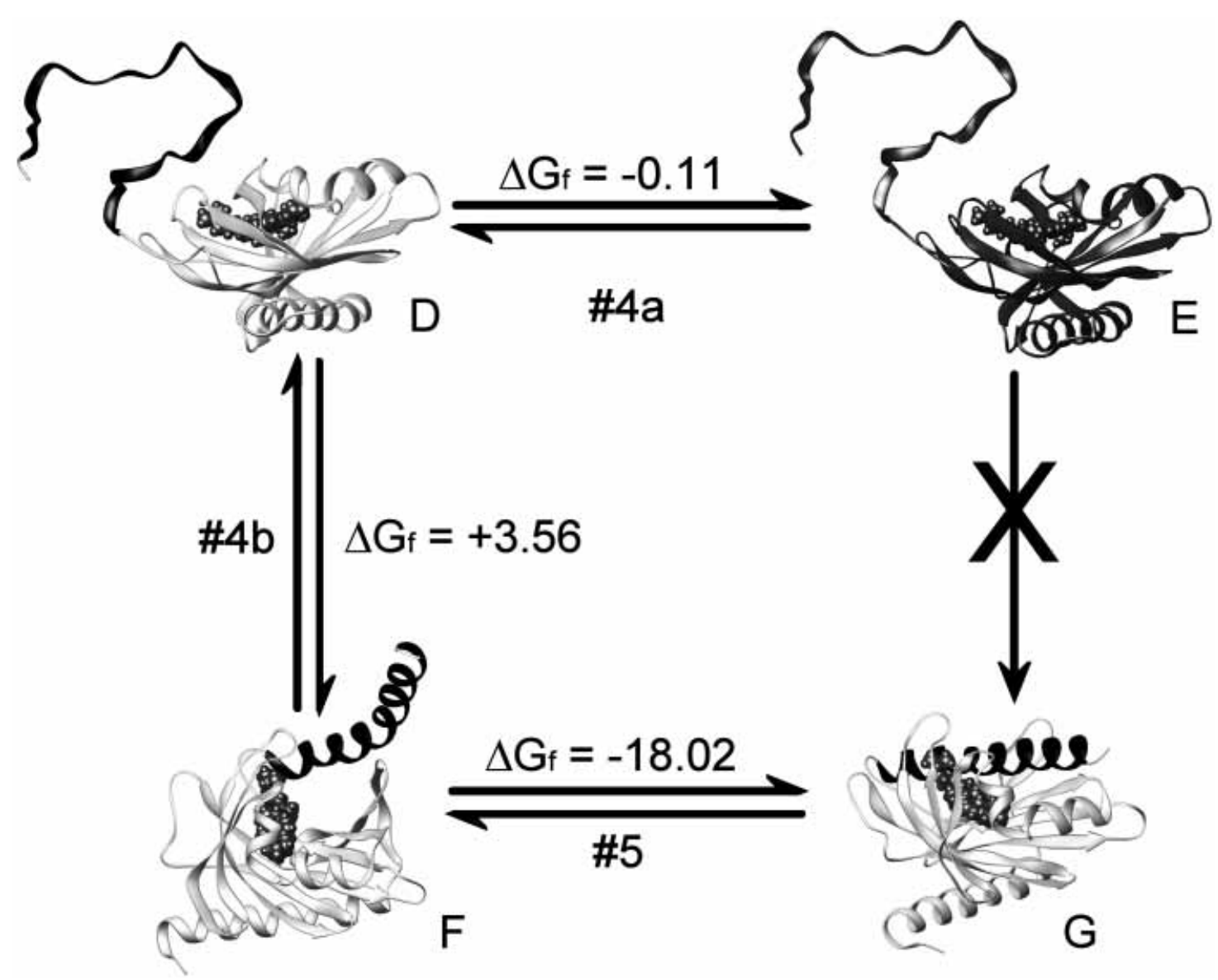

Figure 6 Cholesterol solvation by StAR. Closure of the cholesterol-bound StAR active site can take either of two pathways: (1) closure of the loops adjacent the open binding site (loop $\Omega 1$ and the loop between $\beta$-strands 1 and 2), resulting in a partially refolded state where C-terminal $\alpha$-helix refolding is inhibited (step \#4a, D to E to G); and (2) C-terminal $\alpha$-helix refolding in the opposite direction to Fig. 4, resulting in the trapping of cholesterol inside StAR (steps \#4b and \#5, D to $F$ to $G$ ). The color code is the same as for Fig. 5 with the exception of the closed loop partially folded cholesterol-bound StAR (F) colored dark gray entirely, distinguishing it from the partially unfolded cholesterol-bound StAR (D).

optimal cholesterol binding. The first function is clearly demonstrated through cholesterol docking analyses in the presence of one water molecule. After all, the salt-bridge is located opposite the moveable C-terminal $\alpha$-helix at the bottom of the hydrophobic pocket, where it will not move and to which the $3 \beta-\mathrm{OH}$ group of cholesterol can interact as it binds to StAR.

Although molten globular states are possible for StAR, a minimal structure must be maintained to mediate StAR function. Therefore, the salt-bridge can be visualized as a key component to maintain StAR in a conformation such that cholesterol will bind. This is supported by our mutational analyses of the salt-bridge, as well as the presence of a peptidase-resistant domain in the human StAR, which includes this salt-bridge (Bose et al. 1999). In the case where the salt-bridge is replaced by hydrophobic residues of similar volume (hamster E168 L-R187 M), total loss of StAR-dependent cholesterol transfer into mitochondria is obtained as compared with the mock transfections. This is most likely due to non-specific interactions of the hydrophobic amino acids and thus could lead to improper StAR folding altogether (Bose et al. 1996); the residual cholesterol transfer inside mitochondria is StAR-independent (Bose et al. 1996).

Serine185 was thought to be a potential phosphorylation site and was thus changed into an 


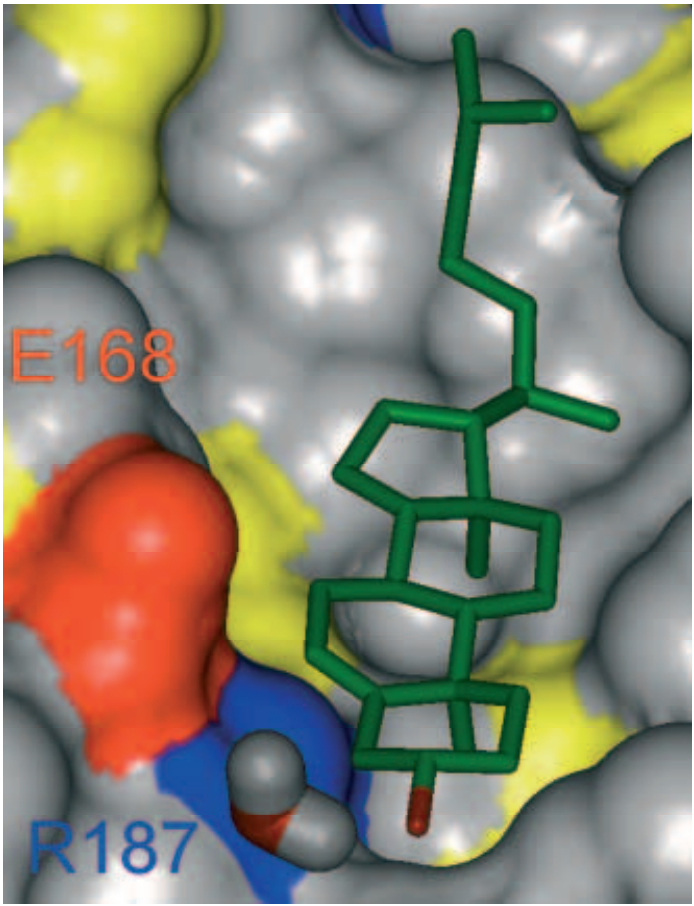

Figure 7 Cholesterol binding inside StAR. Surface diagram of the StAR U-shaped $\beta$-barrel with representation of cholesterol as sticks without hydrogens. One water molecule is depicted as a thick stick, allowing hydrogen bonding of the cholesterol's $3 \beta-\mathrm{OH}$ with Arg187. The color scheme is the same as in Fig. 3B: hydrophobic atoms in gray, potentially polar atoms in yellow, and charged atoms in red (oxygens) and blue (nitrogens).

alanine that cannot be phosphorylated; total loss of StAR-dependent cholesterol transfer was also obtained (Fleury et al., unpublished results). To further test the hypothesis that $\mathrm{S} 185$ is indeed phosphorylated, we mutated S185 into aspartate and glutamate mimicking phosphorylation, and no gain in StAR activity was obtained. The only recuperation of StAR activity was obtained with the mutant S185C, where cysteine greatly resembles serine both in volume and hydrophilicity but still cannot be phosphorylated. Moreover, 2D-PAGE analyses of the S185A mutant did not reveal any differences compared with WT StAR. This clearly indicates that S185 is not phosphorylated and its control on StAR activity comes from structural alterations. Upon a closer look, S185 is located directly beneath the salt-bridge, inaccessible to solvent. It thus becomes clear that not only is the salt-bridge important, but also its immediate environment leading to its proper positioning. In
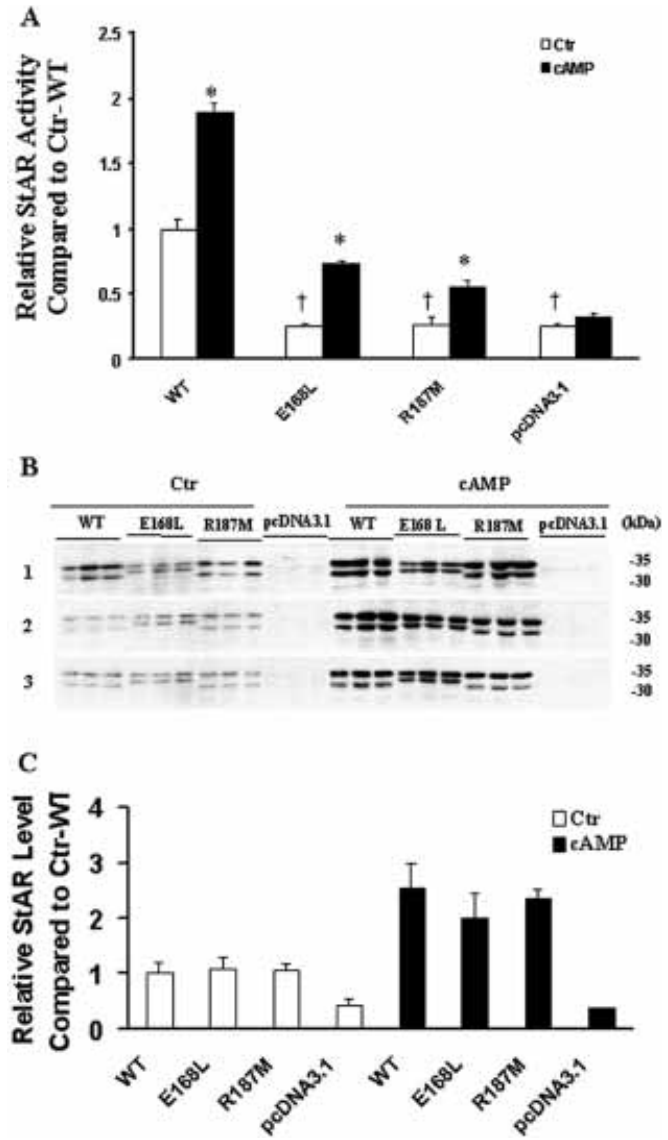

Figure 8 Altered StAR activity of salt-bridge mutants. COS-1 cells co-transfected with F2 and different StAR-pcDNA3.1 plasmids were incubated without (Ctr) and with $(\mathrm{Bu})_{2}$-cAMP (cAMP) for $24 \mathrm{~h}$. (A) Relative StAR activity compared with Ctr-WT; values are the mean of three different experiments. (B) After electrophoresis and transfer on membranes, proteins were revealed using a mouse anti-StAR antibody: results are from three different homogenate preparations. (C) Quantification of the protein bands obtained in B. ${ }^{*} P<0.05$ compared with their own control value; $\dagger P<0.05$ compared with Ctr-WT.

summary, therefore, removal of the salt-bridge by mutagenesis could not only reduce cholesterol affinity to the StAR binding site due to loss of orientation, but could also result in improper folding of StAR altogether as demonstrated for the human StAR mutant E169 G (Bose et al. 1998).

\section{Cholesterol translocation}

The major reason for modeling StAR is to visualize its environment, hopefully leading to an 


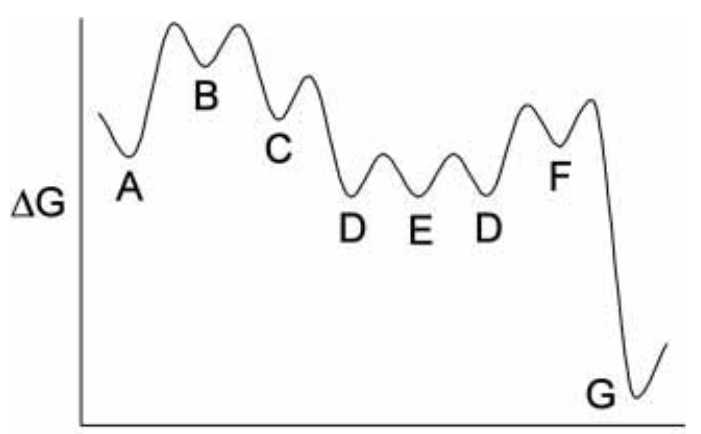

Conformations

Figure 9 Profile of the change in free Gibb's energy. Graphical representation of the results obtained in Figs 4 and 6; the letters represent the same conformations as in these Figures. Under normal conditions, StAR is in equilibrium between to the native and partially unfolded states (A vs C). Approximately $2 \%$ of the StAR population is expected to be in the partially unfolded state. Note that the opened state B should not exist other than to change from the native to the partially unfolded state and vice versa ( $A$ to $C$ and $C$ to $A$ ). Upon cholesterol binding, the cholesterol-bound partially unfolded StAR is slightly more stable than the native state ( $D$ vs $A$ respectively), therefore shifting the equilibrium of the population of StAR to the partially unfolded state. Half of the partially unfolded cholesterolbound StAR population has the loops closed on top of the cholesterol $\left(\mathrm{E} ;\right.$ population ${ }^{\mathrm{D}}=$ population $\left.^{\mathrm{E}}\right)$. The partially refolded StAR (E) can then return to the partially unfolded empty StAR (C) if cholesterol is released, or irreversibly trap cholesterol inside StAR's cholesterol binding site if cholesterol is not released (G). In the latter case, the transient refolded C-terminal $\alpha$-helix with the open binding site $(F)$ is not believed to exist other than to allow the transition from the partially unfolded to native cholesterol-bound StAR. Threshold activation between conformational states are arbitrary and do not represent experimental data. $\Delta G$ is the change in Gibb's free energy sequentially obtained from Figs 4 and 6.

understanding of the cholesterol translocation into mitochondria. At present, two mechanisms are widely accepted: the molten globule, and the intermembrane shuttle. Here, we demonstrate the possibility of structural changes in the hamster StAR supported by theoretical molecular thermodynamics, in accordance with either of these mechanisms.

As one looks at the energy profile of the proposed states of StAR (Fig. 9), two possible mechanisms become clear to account for either the intermembrane shuttle or the molten globular states acting outside mitochondria (Fig. 10). First, in

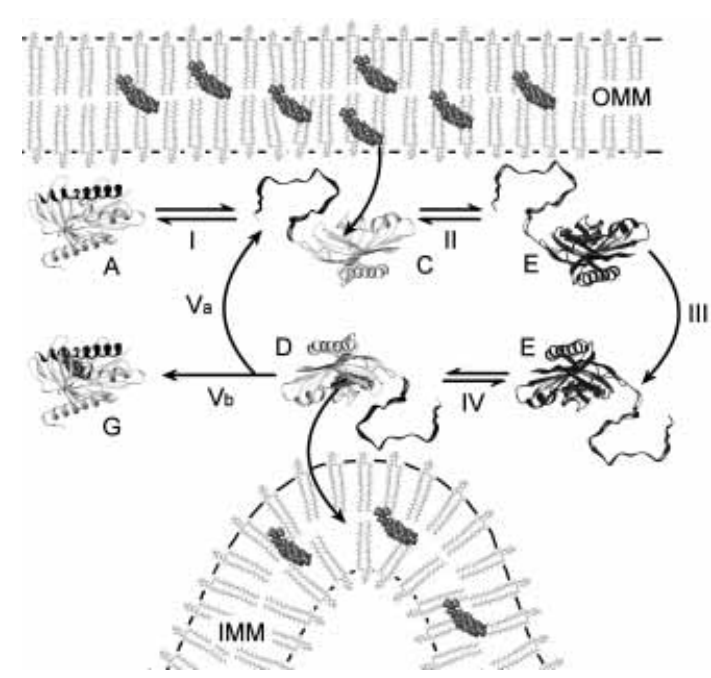

Figure 10 Proposed StAR mechanism in the mitochondrial intermembrane space. Cholesterol is transferred into mitochondria through the flipping of StAR from the outer mitochondrial membrane (OMM) to the inner mitochondrial membrane (IMM). In the intermembrane space at contact sites, the cholesteroldeprived native StAR opens and unfolds through the movement of the C-terminal $\alpha$-helix (step I, A to C). Close to the cholesterol-rich OMM, maybe even interacting with it, cholesterol binds to the exposed StAR binding site and the loops adjacent to the binding site (loop $\Omega 1$ and the loop between $\beta$-strands 1 and 2 ) close, covering cholesterol (step II, C to E). The StAR-cholesterol complex then flips on itself to release cholesterol in the IMM after the loops open to expose cholesterol (steps III and IV, E to D). If cholesterol is released in the IMM, then it can be recycled back to the native/partially unfolded state equilibrium for transport of another cholesterol molecule (step Va, D to C). In the event that no cholesterol gradient exists to drive the transport of cholesterol inside the IMM, the C-terminal $\alpha$-helix of the partially unfolded cholesterol-bound StAR can then refold on top of cholesterol, irreversibly trapping cholesterol inside StAR (step Vb, D to G) and shutting off StAR function; equilibrium between the native and partially unfolded state of StAR is re-established (step I, $A$ to $C)$. The structures, their colors, and their labels are identical to Figs 4 and 6 for consistency.

the mitochondrial intermembrane space, StAR is mainly in the native state (Fig. 9A) in equilibrium with approximately $2 \%$ in the partially unfolded state (Fig. 9G). Upon stimulation (i.e. cAMP), cholesterol is mobilized to the outer mitochondrial membrane, increasing the chemical potential between the inner and outer mitochondrial membranes (Jefcoate et al. 1987). This increased chemical gradient should be sufficient to increase 
the amount of cholesterol-bound StAR, shifting the equilibrium between native and partially unfolded StAR states, towards the partially unfolded state (Fig. 10G). This is because the cholesterol-bound partially unfolded StAR is more stable than empty native StAR (Fig. 9G vs 9A). Upon cholesterol binding, StAR partially closes through the movement of the loops (loop $\Omega 1$ and the loop between $\beta$-strands 1 and $2-$ Fig. $5 \mathrm{E}$ ) and maintains the observed loss of helical structure upon cholesterol binding (Petrescu et al. 2001). This partially 'refolded' state (Figs 5, 6 and 9; state E) then rotationally translates on itself (Fig. 10, step III), now positioning the opened cholesterol-bound cavity towards the inner mitochondrial membrane to allow cholesterol to be transferred. Once flipped, StAR releases the cholesterol in the inner mitochondrial membrane and returns to the outer mitochondrial membrane where it can bind another cholesterol molecule for transfer (Fig. 10D). In the event that the cholesterol gradient across the mitochondrial membranes is insufficient to drive the transfer of cholesterol inside mitochondria, refolding of the $\mathrm{C}$-terminal $\alpha$-helix competes with the release of cholesterol and the recycling of StAR. In other words, if the release of cholesterol is disfavored due to a lowered chemical gradient, cholesterol remains bound to StAR long enough due to no net cholesterol transfer inside mitochondria. This allows enough time for the C-terminal $\alpha$-helix to refold on top of cholesterol; it is widely accepted that refolding of a helix is much slower than transient movement of loops in solvent. Yet, it is impossible at the moment to estimate the differences in energy threshold between the refolding of the $\mathrm{C}$-terminal $\alpha$-helix and the recycling of StAR to the native state after cholesterol release (Fig. 6, steps $4 \mathrm{a}$ vs $4 \mathrm{~b}$, and Fig. 10 , steps $\mathrm{Va}$ and $\mathrm{Vb})$. In virtue of the large stabilization of the closed C-terminal $\alpha$-helix on top of the bound cholesterol in StAR (Fig. 9 G), this step can be visualized as a rapid shut off mechanism halting StAR function, as it is irreversible. Even though these various StAR states can be located throughout the mitochondrial intermembrane, this mechanism will only function if in close proximity to 'contact sites'. In support of this, the complete human StAR has been located in the intermembrane space and the inner mitochondrial membrane facing the intermembrane space by immunoelectron microscopy (King

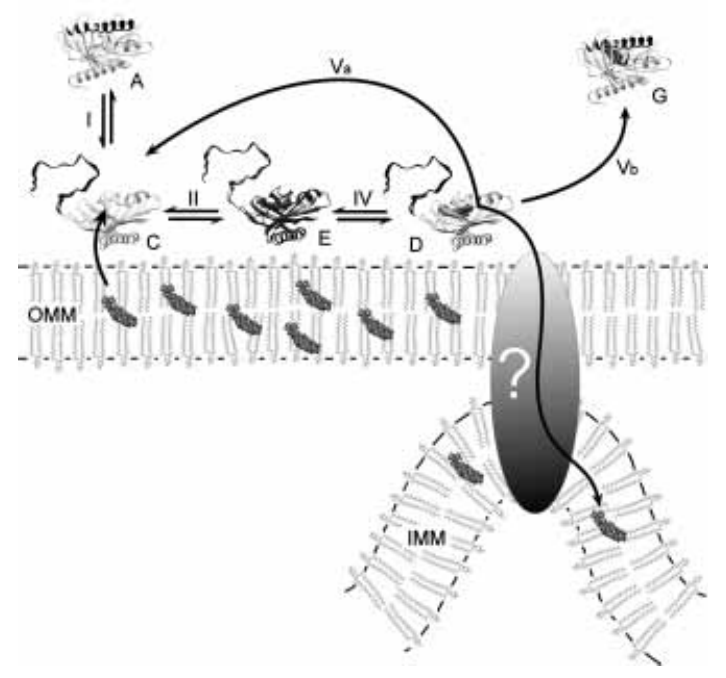

Figure 11 Proposed StAR mechanism in the cytosol. Cholesterol enters mitochondria through the concerted action of a cytosolic StAR with an unidentified factor (?). In this case, StAR unfolding is identical to the case where StAR is located in the mitochondrial intermembrane space (Fig. 10), with the exception that StAR now shuttles cholesterol from the cholesterol-rich outer mitochondrial membrane to the unidentified mitochondrial factor. StAR supposedly remains outside the mitochondria until it is marked for degradation inside the mitochondria. For consistency, the structures, their colors, and their labels are identical to Figs 4, 6, and 10. OMM, outer mitochondrial membrane; IMM, inner mitochondrial membrane.

et al. 1995), and at mitochondrial contact sites by immunoblotting (Cherradi et al. 1997).

Since our STG calculations have been performed in an aqueous solvent, these StAR partially unfolded states are also applicable to StAR in the cytosol (Bose et al. 2002). In this case, StAR functions similarly to that of the intermembrane mechanism, with the exception that StAR does not rotationally translate upon itself. On the other hand, it would seem that StAR needs another unidentified factor to transfer cholesterol inside mitochondria as it does not enter mitochondria itself other than to be degraded. Recently, it has been shown that the peripheral benzodiazepine receptor $(\mathrm{PBR})$ is located preferentially at approximately $70 \AA$ from StAR on the mitochondrial cytosolic surface (West et al. 2001), suggestive of a close interaction for cholesterol transfer inside mitochondria. Therefore, cytosolic StAR mediates cholesterol transfer inside mitochondria 
by 'shuttling' cholesterol from the cholesterol-rich outer mitochondrial membrane to another factor (i.e. PBR) for subsequent cholesterol transfer inside mitochondria (Fig. 11); this mechanism may still be dependent on the cholesterol gradient across the outer and inner mitochondrial membranes. In this case, moreover, it is not inconceivable that cholesterol may be fed to StAR directly from the cytosol cholesterol carriers, such as the sterol carrier protein-2 (SCP-2) (Gallegos et al. 2001).

\section{The pros and cons}

The greatest downfall of our mechanism is the fact that the first 65 amino acids are unaccounted for; this N-terminal region is presently surrounded by controversy. On the one hand, it permits cholesterol transfer in the desorption mechanism (Kallen et al. 1998). On the other hand, it has absolutely nothing to do with cholesterol transfer (Arakane et al. 1996, 1998, Tsujishita \& Hurley 2000). Yet not too long ago, it was demonstrated by 2D-PAGE and 2-phased analyses of cholesterol metabolism, that newly synthesized complete StAR is rapidly processed to the $\mathrm{N}$-terminally cleaved StAR, the form that mediates cholesterol access to P450 scc (Artemenko et al. 2001).

Several observations - that removal of the first 46 N-terminal amino acids only slightly affected protein concentrations and not StAR activity (Fleury et al., unpublished results), that N-terminally cleaved StAR is the active form of StAR mediating cholesterol access to P450 scc (Artemenko et al. 2001), and that through modeling we can justify the existence of molten globular StAR states - further support our mechanism. Therefore, in the case of the intermembrane shuttle, an import sequence-independent internalization of StAR into the mitochondrial intermembrane space is necessary and could be possible (Derman et al. 1993). In the event where StAR functions outside mitochondria, StAR interactions with mitochondrial proteins are key for proper StAR function and still remain to be clearly identified.

Petrescu et al. (2001) have recently observed four novel characteristics of StAR lacking the first 62 amino acids: (1) NBD-cholesterol was bound at two distinct sites, (2) loss of StAR $\alpha$-helical structure occurred when bound with NBD-cholesterol, (3) an average $K_{\mathrm{d}}$ of about $32 \mathrm{nM}$ was seen for both binding sites, and (4) NBD-cholesterol was bound inside the StAR 'tunnel'. First, the authors suggest that there is room inside the 'tunnel' for two NBD-cholesterol molecules. Following our modeling, the replacement of the tunnel by the cavity restrains its volume to permit only one cholesterol molecule. In addition, this is contradictory to previous results showing molar cholesterol binding to the human MLN64 and StAR (Tsujishita \& Hurley 2000). Therefore the second 'cholesterol' binding site seems to be an artifact from the intrinsic polar properties of the NBD-cholesterol chromophoric side-chain.

Secondly, a nanomolar $K_{\mathrm{d}}$ is unlikely for StAR. Although this strong association of 'cholesterol' to StAR could explain the dissociation of cholesterol from the outer mitochondrial membrane as Petrescu et al. (2001) suggested, it cannot explain how cholesterol is released to the inner mitochondrial membrane. Furthermore, such strong interactions are against a rapid cholesterol transfer, as they would slow down StAR efficiency. On the other hand, a $K_{\mathrm{d}}$ in micromolar proportions, such as our theoretical calculated $K_{\mathrm{d}} \sim 95 \mu \mathrm{M}$ validated by STC, supports a rapid release of cholesterol by StAR.

Our STG calculations can account for several key points in the StAR-dependent cholesterol mechanism. First, the N-terminal mitochondrial sequence is not required for cholesterol transfer (Arakane et al. 1996, 1998) either inside the intermembrane space or outside mitochondria in the cytosol. Secondly, a significant population of molten globular StAR is possible (Bose et al. 1999, Christensen et al. 2001) under normal conditions for both mechanisms. Thirdly, the hydrophobic tunnel is nonexistent but rather is replaced by a hydrophobic pocket that opens through the molten globule; according to the MLN64 crystal, it was argued that the openings were too small to allow cholesterol to pass though the hydrophobic tunnel (Tsujishita \& Hurley 2000). Fourthly, the intermembrane shuttle becomes extremely rapid for the transfer of a great quantity of cholesterol in a short space of time (Artemenko et al. 2001) through partially unfolded states of StAR. Fifthly, there is loss of StAR helical structure upon 'cholesterol' binding (Petrescu et al. 2001). Sixthly, the C-terminal region of StAR is critical for StAR activity (Lin et al. 1995, Bose et al. 1996). Seventhly, shutting off StAR-dependent cholesterol transfer inside mitochondria by irreversibly trapping cholesterol inside StAR is in agreement with the 
dependence of cholesterol transfer on newly synthesized StAR (Artemenko et al. 2001). Finally, our proposed StAR unfolding is entirely consistent with similar C-terminal movement and unfolding of PITP $\alpha$, discovered by crystallization, to allow substrate binding (Schouten et al. 2002).

In conclusion, we were able to enlarge our understanding of the elusive StAR-dependent cholesterol transfer into mitochondria through molecular modeling and structure-based thermodynamics. This is supported by our experimental and validated computational (Freire 1993) results presented here, and those of others (Lin et al. 1995). It is now possible to envision a global mechanism accounting for previously contradicting results.

\section{Acknowledgements}

We would like to thank Dr Dale Buchanan Hales (University of Illinois at Chicago) for the anti-StAR antibody and Dr Walter L Miller (University of California at San Francisco) for the F2 construct.

This work was supported by grants from the Canadian Institutes of Health Research and the Heart and Stroke Foundation of Canada to J-G L, and from the Natural Sciences and Engineering Research Council of Canada to P L; J-G L is chercheur boursier de carrière du Fonds de la Recherche en Santé du Québec and P L is chercheur-stratégique du Fonds pour la Formation de Chercheurs et l'Aide à la Recherche.

\section{References}

Alberta JA, Epstein LF, Pon LA \& Orme-Johnson NR 1989 Mitochondrial localization of a phosphoprotein that rapidly accumulates in adrenal cortex cells exposed to adrenocorticotropic hormone or to cAMP. Fournal of Biological Chemistry $\mathbf{2 6 4}$ 2368-2372.

Arakane F, Sugawara T, Nishino H, Liu Z, Holt JA, Pain D, Stocco DM, Miller WL \& Strauss JF III 1996 Steroidogenic acute regulatory protein (StAR) retains activity in the absence of its mitochondrial import sequence: implications for the mechanism of StAR action. [see comments]. PNAS 93 13731-13736.

Arakane F, Kallen CB, Watari H, Foster JA, Sepuri NB, Pain D, Stayrook SE, Lewis M, Gerton GL \& Strauss JF III 1998 The mechanism of action of steroidogenic acute regulatory protein (StAR). StAR acts on the outside of mitochondria to stimulate steroidogenesis. Fournal of Biological Chemistry 273 16339-16345.

Artemenko IP, Zhao D, Hales DB, Hales KH \& Jefcoate CR 2001 Mitochondrial processing of newly synthesized StAR, but not total StAR, mediates cholesterol transfer to $\mathrm{P} 450 \mathrm{scc}$ in adrenal cells. Fournal of Biological Chemistry 276 46583-46596.
Baker BM \& Murphy KP 1998 Prediction of binding energetics from structure using empirical parameterization. Methods in Enzymology 295 294-315.

Bieche I, Tomasetto C, Regnier CH, Moog-Lutz C, Rio MC \& Lidereau R 1996 Two distinct amplified regions at 17q11-q21 involved in human primary breast cancer. Cancer Research $\mathbf{5 6}$ 3886-3890.

Bose HS, Sugawara T, Strauss JF III \& Miller WL 1996 The pathophysiology and genetics of congenital lipoid adrenal hyperplasia. International Congenital Lipoid Adrenal Hyperplasia Consortium. New England Foumal of Medicine 335 1870-1878.

Bose HS, Baldwin MA \& Miller WL 1998 Incorrect folding of steroidogenic acute regulatory protein (StAR) in congenital lipoid adrenal hyperplasia. Biochemistry 37 9768-9775.

Bose HS, Whittal RM, Baldwin MA \& Miller WL 1999 The active form of the steroidogenic acute regulatory protein, StAR, appears to be a molten globule. PNAS 96 7250-7255.

Bose H, Lingappa VR \& Miller WL 2002 Rapid regulation of steroidogenesis by mitochondrial protein import. Nature $\mathbf{4 1 7}$ 87-91.

Brownie AC, Simpson ER, Jefcoate CR, Boyd GS, Orme-Johnson WH \& Beinert H 1972 Effect of ACTH on cholesterol side-chain cleavage in rat adrenal mitochondria. Biochemical and Biophysical Research Communications 46 483-490.

Caron KM, Soo SC, Wetsel WC, Stocco DM, Clark BJ \& Parker KL 1997 Targeted disruption of the mouse gene encoding steroidogenic acute regulatory protein provides insights into congenital lipoid adrenal hyperplasia. PNAS 94 11540-11545.

Cherradi N, Rossier MF, Vallotton MB, Timberg R, Friedberg I, Orly J, Wang XJ, Stocco DM \& Capponi AM 1997 Submitochondrial distribution of three key steroidogenic proteins (steroidogenic acute regulatory protein and cytochrome p450 scc and 3 beta-hydroxysteroid dehydrogenase isomerase enzymes) upon stimulation by intracellular calcium in adrenal glomerulosa cells. Fournal of Biological Chemistry 272 7899-7907.

Christensen K, Bose HS, Harris FM, Miller WL \& Bell JD 2001 Binding of steroidogenic acute regulatory protein to synthetic membranes suggests an active molten globule. Fournal of Biological Chemistry 276 17044-17051.

Clark BJ, Wells J, King SR \& Stocco DM 1994 The purification, cloning, and expression of a novel luteinizing hormone-induced mitochondrial protein in MA-10 mouse Leydig tumor cells. Characterization of the steroidogenic acute regulatory protein (StAR). Fournal of Biological Chemistry $26928314-28322$.

Clark BJ, Combs R, Hales KH, Hales DB \& Stocco DM 1997 Inhibition of transcription affects synthesis of steroidogenic acute regulatory protein and steroidogenesis in MA-10 mouse Leydig tumor cells. Endocrinology 138 4893-4901.

Cockcroft S 1999 Mammalian phosphatidylinositol transfer proteins: emerging roles in signal transduction and vesicular traffic. Chemistry and Physics of Lipids 98 23-33.

Creighton TE 1993 Proteins in solution and in membranes. In Proteins: Structure and Molecular Properties, edn 2, pp 261-328. New York: W. H. Freeman and Co.

Crivello JF \& Jefcoate CR 1980 Intracellular movement of cholesterol in rat adrenal cells. Kinetics and effects of inhibitors. Fournal of Biological Chemistry 255 8144-8151.

Cunningham E, Thomas GM, Ball A, Hiles I \& Cockcroft S 1995 Phosphatidylinositol transfer protein dictates the rate of inositol trisphosphate production by promoting the synthesis of PIP2. Current Biology 5 775-783.

Dauber-Osguthorpe P, Roberts VA, Osguthorpe DJ, Wolff J, Genest M \& Hagler AT 1988 Structure and energetics of ligand binding to proteins: $E$. coli dihydrofolate reductase-trimethoprim, a drug-receptor system. Proteins: Structure, Function and Genetics 4 $31-47$. 
Derman AI, Puziss JW, Bassford PJ Jr \& Beckwith J 1993 A signal sequence is not required for protein export in prlA mutants of Escherichia coli. EMBO Fournal 12 879-888.

Epstein LF \& Orme-Johnson NR 1991 a Acute action of luteinizing hormone on mouse Leydig cells: accumulation of mitochondrial phosphoproteins and stimulation of testosterone synthesis. Molecular and Cellular Endocrinology 81 113-126.

Epstein LF \& Orme-Johnson NR $1991 b$ Regulation of steroid hormone biosynthesis. Identification of precursors of a phosphoprotein targeted to the mitochondrion in stimulated rat adrenal cortex cells. Fournal of Biological Chemistry 266 19739-19745.

Farkash Y, Timberg R \& Orly J 1986 Preparation of antiserum to rat cytochrome P-450 cholesterol side chain cleavage, and its use for ultrastructural localization of the immunoreactive enzyme by protein A-gold technique. Endocrinology 118 1353-1365.

Fleury A, Mathieu AP, Ducharme L, Hales DB \& Le Hou JG, unpublished results.

Freire E 1993 Structural thermodynamics: prediction of protein stability and protein binding affinities. Archives of Biochemistry and Biophysics 303 181-184.

Gallegos AM, Atshaves BP, Storey SM, Starodub O, Petrescu AD, Huang H, McIntosh AL, Martin GG, Chao H, Kier AB \& Schroeder F 2001 Gene structure, intracellular localization, and functional roles of sterol carrier protein-2. Progress in Lipid Research $40498-563$.

Harikrishna JA, Black SM, Szklarz GD \& Miller WL 1993 Construction and function of fusion enzymes of the human cytochrome P450 scc system. DNA and Cell Biology 12 371-379.

Hartigan JA, Green EG, Mortensen RM, Menachery A, Williams GH \& Orme-Johnson NR 1995 Comparison of protein phosphorylation patterns produced in adrenal cells by activation of cAMP-dependent protein kinase and Ca-dependent protein kinase. Fournal of Steroid Biochemistry and Molecular Biology $\mathbf{5 3}$ 95-101.

Hilser VJ \& Freire E 1997 Predicting the equilibrium protein folding pathway: structure-based analysis of staphylococcal nuclease. Proteins 27 171-183.

Homma Y \& Emori Y 1995 A dual functional signal mediator showing RhoGAP and phospholipase C-delta stimulating activities. EMBO Fournal 14 286-291.

Jefcoate CR, DiBartolomeis MJ, Williams CA \& McNamara BC 1987 ACTH regulation of cholesterol movement in isolated adrenal cells. Fournal of Steroid Biochemistry 27 721-729.

Kallen CB, Billheimer JT, Summers SA, Stayrook SE, Lewis M \& Strauss JF III 1998 Steroidogenic acute regulatory protein (StAR) is a sterol transfer protein. Fournal of Biological Chemistry 273 26285-26288.

King SR, Ronen-Fuhrmann T, Timberg R, Clark BJ, Orly J \& Stocco DM 1995 Steroid production after in vitro transcription, translation, and mitochondrial processing of protein products of complementary deoxyribonucleic acid for steroidogenic acute regulatory protein. Endocrinology 136 5165-5176.

King SR, Liu Z, Soh J, Eimerl S, Orly J \& Stocco DM 1999 Effects of disruption of the mitochondrial electrochemical gradient on steroidogenesis and the steroidogenic acute regulatory (StAR) protein. Fournal of Steroid Biochemistry and Molecular Biology 69 $143-154$.

Laemmli UK 1970 Cleavage of structural proteins during the assembly of the head of bacteriophage TH. Nature 227 680-685.

Laskowski RA, MacArthur MW, Hutchinson EG \& Thornton JM 1993 PROCHECK: a program to check the stereochemical quality of protein structures. Fournal of Applied Crystallography 26 283-291.

Lavigne P, Bagu JR, Boyko R, Willard L, Holmes CF \& Sykes BD 2000 Structure-based thermodynamic analysis of the dissociation of protein phosphatase-1 catalytic subunit and microcystin-LR docked complexes. Protein Science 9 252-264.

LeHoux J-G, Mason JI \& Ducharme L 1992 In vivo effects of adrenocorticotropin on hamster adrenal steroidogenic enzymes. Endocrinology 131 1874-1882.

LeHoux J-G, Lefebvre A, Ducharme L, Lehoux J, Martel D \& Brière N 1996 Some effects of a low sodium intake on the expression of $\mathrm{P} 450$ aldosterone synthase in the hamster adrenal cortex: immunoblotting, immunofluorescent and immuno-gold electron microscopic studies. Fournal of Endocrinology 149 341-349.

Lin D, Sugawara T, Strauss JF III, Clark BJ, Stocco DM, Saenger P, Rogol A \& Miller WL 1995 Role of steroidogenic acute regulatory protein in adrenal and gonadal steroidogenesis. [see comments]. Science 267 1828-1831.

Masucci JD, Rerie WG, Foreman DR, Zhang M, Galway ME, Marks MD \& Schiefelbein JW 1996 The homeobox gene GLABRA2 is required for position-dependent cell differentiation in the root epidermis of Arabidopsis thaliana. Development 122 1253-1260

Moog-Lutz C, Tomasetto C, Regnier CH, Wendling C, Lutz Y, Muller D, Chenard MP, Basset P \& Rio MC 1997 MLN64 exhibits homology with the steroidogenic acute regulatory protein (StAR) and is over-expressed in human breast carcinomas. International Fournal of Cancer 71 183-191.

Petrescu AD, Gallegos AM, Okamura Y, Strauss JF \& Schroeder F 2001 Steroidogenic acute regulatory protein binds cholesterol and modulates mitochondrial membrane sterol domain dynamics. Fournal of Biological Chemistry 276 36970-36982.

Pon LA \& Orme-Johnson NR 1988 Acute stimulation of corpus luteum cells by gonadotrophin or adenosine $3^{\prime}, 5^{\prime}$-monophosphate causes accumulation of a phosphoprotein concurrent with acceleration of steroid synthesis. Endocrinology 123 1942-1948.

Pon LA, Hartigan JA \& Orme-Johnson NR 1986 Acute ACTH regulation of adrenal corticosteroid biosynthesis. Rapid accumulation of a phosphoprotein. Fournal of Biological Chemistry 261 13309-13316.

Pon L, Moll T, Vestweber D, Marshallsay B \& Schatz G 1989 Protein import into mitochondria: ATP-dependent protein translocation activity in a submitochondrial fraction enriched in membrane contact sites and specific proteins. Fournal of Cell Biology 109 2603-2616.

Ponting CP \& Aravind L 1999 START: a lipid-binding domain in StAR, HD-ZIP and signalling proteins. Trends in Biochemical Sciences 24 130-132.

Ponting CP, Schultz J, Milpetz F \& Bork P 1999 SMART: identification and annotation of domains from signalling and extracellular protein sequences. Nucleic Acids Research 27 229-232.

Privalle CT, Crivello JF \& Jefcoate CR 1983 Regulation of intramitochondrial cholesterol transfer to side-chain cleavage cytochrome P-450 in rat adrenal gland. PNAS 80 702-706.

Raya A, Revert F, Navarro S \& Saus J 1999 Characterization of a novel type of serine/threonine kinase that specifically phosphorylates the human goodpasture antigen. Fournal of Biological Chemistry 274 12642-12649.

Schouten A, Agianian B, Westerman J, Kroon J, Wirtz KW \& Gros P 2002 Structure of apo-phosphatidylinositol transfer protein alpha provides insight into membrane association. EMBO $\mathcal{7} 21$ 2117-2121.

Schwaiger M, Herzog V \& Neupert W 1987 Characterization of translocation contact sites involved in the import of mitochondrial proteins. Fournal of Cell Biology 105 235-246.

Simpson ER, McCarthy JL \& Peterson JA 1978 Evidence that the cycloheximide-sensitive site of adrenocorticotropic hormone action is in the mitochondrion. Changes in pregnenolone formation, cholesterol content, and the electron paramagnetic resonance spectra of cytochrome P-450. Fournal of Biological Chemistry 253 3135-3139. 
Stocco DM 2000 Intramitochondrial cholesterol transfer. Biochimica et Biophysica Acta 1486 184-197.

Stocco DM 2001 Tracking the role of a star in the sky of the new millennium. Molecular Endocrinology 15 1245-1254.

Stocco DM \& Clark BJ 1996 Regulation of the acute production of steroids in steroidogenic cells. Endocrine Revieves 17 221-244.

Sugawara T, Holt JA, Driscoll D, Strauss JF III, Lin D, Miller WL, Patterson D, Clancy KP, Hart IM \& Clark BJ 1995 Human steroidogenic acute regulatory protein: functional activity in COS-1 cells, tissue-specific expression, and mapping of the structural gene to $8 \mathrm{p} 11.2$ and a pseudogene to chromosome 13 . PNAS 92 4778-4782.

Thompson JD, Higgins DG \& Gibson TJ 1994 CLUSTAL W: improving the sensitivity of progressive multiple sequence alignment through sequence weighting, position-specific gap penalties and weight matrix choice. Nucleic Acids Research 22 4673-4680.
Tsujishita Y \& Hurley JH 2000 Structure and lipid transport mechanism of a StAR-related domain. Nature Structural Biology 7 408-414.

Watari H, Arakane F, Moog-Lutz C, Kallen CB, Tomasetto C, Gerton GL, Rio MC, Baker ME \& Strauss JF III 1997 MLN64 contains a domain with homology to the steroidogenic acute regulatory protein (StAR) that stimulates steroidogenesis. PNAS 94 8462-8467.

West LA, Horvat RD, Roess DA, Barisas BG, Juengel JL \& Niswender GD 2001 Steroidogenic acute regulatory protein and peripheral-type benzodiazepine receptor associate at the mitochondrial membrane. Endocrinology 142 502-505.

Received 14 June 2002

Accepted 9 September 2002 